\title{
Editorial: Recent Advances in Bioremediation/Biodegradation by Extreme Microorganisms
}

\author{
Edgardo Rubén Donati ${ }^{*}$, Rajesh Kumar Sani ${ }^{2}$, Kian Mau Goh ${ }^{3}$ and Kok-Gan Chan ${ }^{4,5}$ \\ ${ }^{1}$ Facultad de Ciencias Exactas, CINDEFI (CCT, La Plata-CONICET, UNLP), Universidad Nacional de la Plata, La Plata, \\ Argentina, ${ }^{2}$ Department of Chemical and Biological Engineering, South Dakota School of Mines and Technology, Rapid City, \\ SD, United States, ${ }^{3}$ Faculty of Sciences, Universiti Teknologi Malaysia, Skudai, Malaysia, ${ }^{4}$ Division of Genetics and Molecular \\ Biology, Faculty of Science, Institute of Biological Sciences, University of Malaya, Kuala Lumpur, Malaysia, ${ }^{5}$ International \\ Genome Centre, Jiangsu University, Zhenjiang, China
}

Keywords: pollutants, bioremediation, biodegradation, biomineralization, extremophiles

\section{Editorial on the Research Topic}

\section{Recent Advances in Bioremediation/Biodegradation by Extreme Microorganisms}

\section{OPEN ACCESS}

Edited by:

Jean Armengaud,

Commissariat à l'Energie Atomique et aux Energies Alternatives

(CEA), France

Reviewed by:

Rafael Bosch

University of the Balearic

Islands, Spain

*Correspondence:

Edgardo Rubén Donat

donati@quimica.unlp.edu.ar

Specialty section:

This article was submitted to Microbiotechnology, Ecotoxicology and Bioremediation,

a section of the journal

Frontiers in Microbiology

Received: 26 June 2019

Accepted: 26 July 2019

Published: 14 August 2019

Citation:

Donati ER, Sani RK, Goh KM and Chan K-G (2019) Editorial: Recent Advances in

Bioremediation/Biodegradation by

Extreme Microorganisms.

Front. Microbiol. 10:1851.

doi: 10.3389/fmicb.2019.01851
To remediate polluted sites, biological processes have many advantages from economic, environmental, and practical aspects. Adsorption and biodegradation of organic contaminants and the immobilization, mobilization, and/or transformation of metal(loid)s are the main remediation processes that can be mediated by the action of several microorganisms especially those extremophiles surviving in hostile environments with high concentrations of pollutants. The aim of this Research Topic of Frontiers in Microbiology is to provide an appropriate platform to publish the latest results on the bioremediation of various pollutants by extremophilic pure cultures or microbial consortia. This Research Topic consists of 4 reviews and 7 original articles.

Marques reviewed the theme of extremophiles as microfactories which are able to provide genetic or metabolic mechanisms as controlled services to the clean-up of environmental pollution. The review focuses on metal and radionuclides pollution, and includes a discussion about the use of synthetic biology to improve the bioremediation processes.

Two articles in this Research Topic are focused on heavy metal(loid)s contaminants. Figueroa et al. described that several microorganisms exhibited high resistance to 19 metal(loid)s. Most of those strains displayed metal or metalloid reducing activity, and have been successfully used for the biological synthesis of nanostructures containing metal(loid)s. Tellurium and gold nanostructures showed antibacterial properties, which inhibited E. coli and L. monocytogenes growth. Acid mine drainage (AMD) is considered a severe environmental problem provoked by the microbial oxidation of sulfidic minerals. Gupta et al. explored the abundance and role of indigenous microorganisms displaying sulfate- and metal(loid)- reducing activity in the natural attenuation of an AMD impacted soil (AIS). The addition of nutrients (e.g., cysteine and lactate) to AIS increased the activity of such microorganisms achieving an increase in $\mathrm{pH}$ from 3.5 to 6.6, and reduction of sulfate (95\%), iron (50\%), and other heavy metals. In this way, Gupta et al. demonstrated that addition of nutrients could biostimulated the growth of some members of phylum Firmicutes (e.g., sulfate- and iron- reducing microorganisms) and bioremediate AMD impacted sites.

Orellana et al. reviewed extensively the most recent research on polyextremophilic microorganisms isolated from a wide range of extreme environments including salars, geothermal springs, deserts, ice fields, and diverse zones in Chile such as Altiplano, Atacama Desert, Central Chile, Patagonia, and Antarctica. This review also discussed the molecular and physiological capabilities of many of these isolates which were beneficial for bioremediation processes. 
Diverse anthropogenic activities, particularly the emission due to the burning of fossil fuels, have triggered an alarming rise of $\mathrm{CO}_{2}$ in the environment. A description of the measures of greenhouse gases emission is reviewed by Bose and Satyanarayana. In this review, authors discussed the merits and demerits of various approaches with extensive bibliographical material. Finally, a deep description of the use of carbonic anhydrases (CA) for biomineralization of $\mathrm{CO}_{2}$ was included. This methodology was proposed as one of the most economical methods to mitigate global warming.

The other six articles in this Research Topic are related to the bioremediation of organic pollutants. Park and Park described the strategies for alkane degradation under extreme conditions (e.g., low and high temperatures, high salt, and acidic and anaerobic conditions). Alkane degraders seem to possess exclusive metabolic pathways and survival strategies. The thermophilic sulfate-reducing archaeon Archaeoglobus fulgidus uses a novel alkylsuccinate synthase for longchain alkane degradation, and the thermophilic Candidatus Syntrophoarchaeum butanivorans anaerobically oxidizes butane via alkyl-coenzyme $M$ formation. In addition to alkane degradation, extremophiles produce energy via the glyoxylate shunt and the Pta-AckA pathway when grown on a diverse range of alkanes under stress conditions.

Blázquez et al. focused on the bioremediation of aromatic compounds such as toluene and xylenes. The degradation of such pollutants is relevant due to their carcinogenic and neurotoxic effects to humans. This article provided evidences that the bss and bbs genes are not only essential for anaerobic degradation of toluene but also for $\mathrm{m}$-xylene oxidation in the beta-proteobacterium Azoarcus sp. The peripheral pathway for the anaerobic oxidation of toluene would consist of an initial activation catalyzed by a benzylsuccinate synthase and a subsequent modified oxidation of benzylsuccinate to benzoylCoA and succinyl-CoA (both pathways encoded by bbs genes). $\mathrm{Su}$ et al. determined the crystal structure of the dibenzothiophene (DBT) sulfone monooxygenase $(B d s A)$ from Bacillus subtilis at the resolution of $2.2 \AA$. This is one of the key enzymes in the $4 \mathrm{~S}$ desulfurization pathway catalyzing the oxidation of DBT sulfone to 2 '-hydroxybiphenyl 2-sulfonic acid. The structure of the BdsA-FMN complex at $2.4 \AA$ was also determined. Finally, $\mathrm{Su}$ et al. showed that mutations in the residues involved in catalysis or flavin substrate-binding, resulted in a significant loss of enzymatic activity.

Meier et al. studies the catabolism of hydroxylated aromatic acids in $A$. adeninivorans and showed that the genes encoding enzymes involved catabolic pathway of gallic acid are induced using aromatic acid substrates as inducers. Through the construction of gallic acid decarboxylase disruption mutants, the authors showed that gallic acid decarboxylase Agdclp was the only enzyme responsible for the transformation of gallic acid. They suggest that this enzyme might have useful industrial applications not only in bioremediation processes but also in synthesis of chemicals.

Chandra et al. detected that effluents discharged from the pulp and paper industry contain various refractory and androgenic compounds, even after secondary treatment by activated processes. Most of these compounds are classified as endocrinedisrupting chemicals and are environmental toxicants. This study also assessed the degradability of such compounds by biostimulation. The results suggested that pulp and paper mill wastewater, after this secondary detoxification process, could be safe for disposal.

Lastly, Kirtzel et al. investigated the ability of Schizophyllum commune to degrade black slates (e.g., metamorphic rocks rich in sulfides, heavy metals and organic matters). S. commune is a filamentous basidiomycete possesses a broad range of enzymes including multicopper oxidases such as laccases and laccaselike oxidases. Both life forms (haploid monokaryotic and mated dikaryotic strains) were able to degrade the slate releasing metals at the same time.

This Research Topic of Frontiers in Microbiology shows how bioremediation by means of extremophiles is an active research theme.

\section{AUTHOR CONTRIBUTIONS}

All authors listed have made a substantial, direct and intellectual contribution to the work, and approved it for publication.

\section{FUNDING}

ED is Superior Researcher at CINDEFI (CONICET, Argentina) and acknowledges the financial support from ANPCYT (PICT 20150463 and PICT 2016 2535). This project was co-financially supported by Universiti Teknologi Malaysia RU grant (Grant number: 16H89) and (UK-SEA-NUOF) with project number 4B297. K-GC thanked University of Malaya for financial support (PPP grants: PG136-2016A, PG133-2016A, HIR grant: H50001-A-000027). RS acknowledges the support from National Science Foundation in the form of BuG ReMeDEE initiative (Award \# 1736255).

Conflict of Interest Statement: The authors declare that the research was conducted in the absence of any commercial or financial relationships that could be construed as a potential conflict of interest.

Copyright (c) 2019 Donati, Sani, Goh and Chan. This is an open-access article distributed under the terms of the Creative Commons Attribution License (CC BY). The use, distribution or reproduction in other forums is permitted, provided the original author(s) and the copyright owner(s) are credited and that the original publication in this journal is cited, in accordance with accepted academic practice. No use, distribution or reproduction is permitted which does not comply with these terms. 